BULL. AUSTRAL. MATH. SOC.

VOL. $14(1976), 1-5$.

\title{
On convergence in capacity
}

\section{Burnett Meyer}

The (logarithmic) capacity or transfinite diameter is originally defined for compact sets in the complex plane. An extension may be made by defining the capacity of a given arbitrary set in the plane as the supremum of the capacities of all compact sets contained in the given set. Convergence in capacity is defined analogously to convergence in measure. It is shown in this paper that properties of convergence in capacity are also analogous to those of convergence in measure.

Recently it has been shown that certain sequences of Padé approximants converge in capacity $[3,4,5]$. The definition of convergence in capacity is analogous to that of convergence in measure. We shall show that the properties of these two kinds of convergence are also analogous.

\section{Preliminaries}

For the definition and properties of the (logarithmic) capacity or transfinite diameter, $C(F)$, of a compact set in the complex plane, see [1, pp. 293-314] or [2, pp. 264-289]. Following Tsuji [8, pp. 53-89] we define as follows the capacity of an arbitrary set $S$ in the complex plane:

$$
C(S)=\sup _{F \in F} C(F)
$$

where $F$ is the set of all compact sets $F$ contained in $S . C(S)$ is cometimes called the inner capacity of $S$, since it is defined analogously to the inner Lebesgue measure. (An outer capacity can also be defined, but we shall not use it.) A property which holds everywhere except on a set of

Received 1 October 1975. 
capacity zero will be said to hold approximately everywhere.

An important property of capacity is given in the following theorem.

Let $S_{n}(n=1,2, \ldots)$ be Borel sets contained in $|z| \leq R$, and Let $S=\bigcup_{n=1}^{\infty} S_{n}$. Then

$$
\{\log [2 R / C(S)]\}^{-1} \leq \sum_{n=1}^{\infty}\left\{\log \left[2 R / C\left(S_{n}\right)\right]\right\}^{-1}
$$

This is an easy generalization of Theorem III.17 on page 63 of [8].

2. Definition and properties of convergence in capacity

DEFINITION. Let $f_{n}(n=1,2, \ldots)$ be complex Borel-measurable functions on a bounded domain $D$. The sequence $\left\{f_{n}\right\}$ is said to converge in capacity to a Borel-measurable function $f$ on $D$ if and only if for every $\varepsilon>0, \lim _{n \rightarrow \infty} C\left(\left\{z \in D:\left|f_{n}(z)-f(z)\right| \geq \varepsilon\right\}\right)=0$. The sequence $\left\{f_{n}\right\}$ is said to be a Cauchy sequence in capacity on $D$ if and only if for every $\varepsilon>0, \lim _{m, n \rightarrow \infty} C\left(\left\{z \in D:\left|f_{n}(z)-f_{m}(z)\right| \geq \varepsilon\right\}\right)=0$.

The collection of complex Borel-measurable functions is closed under addition, multiplication, and pointwise limits. Padé approximants, being meromorphic in any bounded domain, are Borel-measurable functions.

Convergence in capacity has properties which are analogous to those of convergence in measure, as is seen in the following theorems.

THEOREM 1. Let $f, g, f_{1}, f_{2}, \ldots$ be complex Borel-measurable functions on a bounded domain $D$, each function finite approximately everywhere.

(a) If $\left\{f_{n}\right\}$ converges to $f$ in capacity on $D$ and $f(z)=g(z)$ approximately everywhere on $D$, then $\left\{f_{n}\right\}$ converges to $g$ in capacity on $D$.

(b) If $\left\{f_{n}\right\}$ converges to $f$ in capacity on $D$ and $\left\{f_{n}\right\}$ converges to $g$ in capacity on $D$, then $f(z)=g(z)$ approximately 
everywhere on $D$.

(c) If $\left\{f_{n}\right\}$ converges to $f$ in capacity on $D$, then $\left\{f_{n}\right\}$ is a Cauchy sequence in capacity on $D$.

The proof is omitted, since it is similar to the proof of the analogous theorem for convergence in measure [7, pp. 265-268].

THEOREM 2. Let $D$ be a bounded domain and $\left\{f_{n}\right\}$ a Cauchy sequence in capacity of Borel-measurable functions, finite approximately everywhere on $D$. Then there exists a Borel-measurable function $f$, which is finite everywhere on $D$, such that $\left\{f_{n}\right\}$ converges to $f$ in capacity and such that a subsequence of $\left\{f_{n}(z)\right\}$ converges to $f(z)$ approximately everywhere on $D$. For each $\varepsilon>0$ there exists a set $E \subset D$ such that $C(E)<\varepsilon$ and such that a subsequence of $\left\{f_{n}(z)\right\}$ converges uniformly on $D-E^{\prime}$.

Proof. Let $D \subset\{z:|z| \leq R\}$. Choose integers $N_{j}(j=1,2, \ldots)$ such that

$$
C\left\{\left\{z \in D:\left|f_{n}(z)-f_{m}(z)\right| \geq 2^{-j}\right\}\right)<2 R \exp \left(-e^{j}\right),
$$

for $m \geq N_{j}, n \geq N_{j}$. Let $g_{j}=f_{N_{j}}$,

$$
G_{j}=\left\{z \in D:\left|g_{j}(z)-g_{j+1}(z)\right| \geq 2^{-j}\right\}
$$

Then $C\left(G_{j}\right)<2 R \exp \left(-e^{j}\right)$. Let $X_{0}=\left\{z \in D: f_{n}(z)=\infty\right.$ for some $\left.n\right\}$; then $C\left(x_{0}\right)=0$.

$$
\begin{aligned}
& \text { Let } E_{k}=X_{0} \cup \bigcup_{j=k}^{\infty} G_{j}(k=1,2, \ldots) . \text { Then, by }(1), \\
& \begin{aligned}
\left\{\log \left[2 R / C\left(E_{k}\right)\right]\right\}^{-1} & \leq\left\{\log \left[2 R / C\left(X_{0}\right)\right]\right\}^{-1}+\sum_{j=k}^{\infty}\left\{\log \left[2 R / C\left(G_{j}\right)\right]\right\}^{-1} \\
& =0+\sum_{j=k}^{\infty} e^{-j}<1 / e^{k-1} .
\end{aligned}
\end{aligned}
$$

From this it follows that $C\left(E_{k}\right)<2 R \exp \left(-e^{k-1}\right)$, and $C\left(E_{k}\right) \rightarrow 0$ as 


$$
\begin{gathered}
\text { Now suppose } z\left(D-E_{k} \text {. Then } z \in \prod_{j=k}^{\infty}\left(D-G_{j}\right)\right. \text {, and } \\
\left|g_{j}(z)-g_{j+1}(z)\right|<2^{-j}(j=1,2, \ldots) \text {. Hence, if } k \leq j<m, \\
\left|g_{j}(z)-g_{m}(z)\right| \leq \sum_{n=j}^{m-1}\left|g_{n}(z)-g_{n+1}(z)\right|<\sum_{n=j}^{\infty} 2^{-n}=2^{-j+1} .
\end{gathered}
$$

Thus, the sequence $\left\{g_{j}(z)\right\}$ is uniformly convergent (to a finite limit) on $D-E_{k}$.

Let $F=\prod_{k=1}^{\infty} E_{k}$. Since $C(F) \leq C\left(E_{k}\right)$ for every $k, C(F)$ must be zero. We see that $\left\{g_{j}(z)\right\}$ converges to a finite limit for $z \in D-F$ because $D-F=\bigcup_{k=1}^{\infty}\left(D-E_{k}\right)$. Thus $\left\{g_{n}\right\}$ converges approximately everywhere on $D$.

Let $f(z)=\lim g_{n}(z)$ for $z \in D-F, f(z)=0$ for $z \in F$. The function $f$ is Borel-measurable and is finite everywhere on $D[6$, pp. 11-14]. We wish to prove that $\left\{f_{n}\right\}$ converges to $f$ in capacity. Let $\varepsilon>0$ and $\delta>0$. Choose $k$ so that $c\left(E_{k}\right)<\delta$, and choose $m$ so that $\left|g_{j}(z)-f(z)\right|<\varepsilon$ for $j \geq m, z \in D-E_{k}$. Then, if $j \geq m$,

$$
C\left(\left\{z \in D:\left|g_{j}(z)-f(z)\right| \geq \varepsilon\right\}\right) \leq C\left(E_{k}\right)<\delta .
$$

Hence $\left\{g_{j}\right\}$ converges to $f$ in capacity.

$$
\begin{array}{r}
\text { Since }\left|f_{n}(z)-f(z)\right| \leq\left|f_{n}(z)-g_{j}(z)\right|+\left|g_{j}(z)-f(z)\right| \\
\left\{z \in D:\left|f_{n}(z)-f(z)\right| \geq \varepsilon\right\} \subset\left\{z \in D:\left|f_{n}(z)-g_{j}(z)\right| \geq \varepsilon / 2\right\} \cup \\
\left\{z \in D:\left|g_{j}(z)-f(z)\right| \geq \varepsilon / 2\right\} .
\end{array}
$$

By choosing $n$ and $j$ sufficiently large, the capacity of each of the sets on the right may be made arbitrarily small. Applying (1), we conclude that $\left\{f_{n}\right\}$ converges to $f$ in capacity on $D$. This completes the proof of 
Theorem 2.

\section{References}

[1] G.M. Goluzin, Geometric theory of functions of a complex variable (Translations of Mathematical Monographs, 26. Amer. Math. Soc., Providence, Rhode Island, 1969).

[2] Einar Hille, Analytic function theory, Vol. II (Ginn, Boston, New York, Chicago, Atlanta, Dallas, Palo Alto, Toronto, 1962).

[3] William B. Jones and W.J. Thron, "On the convergence of Padé approximants", SIAM J. Math. AnaZ. 6 (1975), 9-16.

[4] J. Nuttall, "The convergence of Padé approximants of mexomorphic functions", J. Math. Anal. Appl. 31 (1970), 147-153.

[5] Ch. Pommerenke, "Padé approximants and convergence in capacity", $J$. Math. Anal. App Z. 41 (1973), 775-780.

[6] Walter Rudin, Real and complex conalysis (McGraw-Hill, New York, St. Louis, San Francisco, Toronto, London, Sydney, 1966).

[7] Angus E. Taylor, General theory of functions and integration (Blaisdell, New York, Toronto, London, 1965).

[8] M. Tsuji, Potential theory in moderm function theory (Maruzen, Tokyo, 1959).

Department of Mathematics,

University of Colorado,

Boulder,

Colorado,

USA. 